\title{
EL VALOR DE LA PAZ, UN DESTINO QUE ALCANZAR
}

Esther Fragoso Fernández*

¿Qué grandes conquistas ha hecho el hombre? Posee el fuego, pero no ha podido escapar a su furia... Domestica las plantas, pero no ha logrado saciar su hambre... Levanta ciudades, pero ha caído en las calles...

Construye máquinas, pero ha destruido la naturaleza...

Tiende puentes, pero ha quedado incomunicado... Explora el espacio, pero no se conoce....

Prolonga su vida, pero está solo... Enciende luces en las noches, pero está ciego...

Alberto Morales Damián

\section{Resumen:}

La paz más que un valor buscado por el ser humano es una situación anhelada en la humanidad, sin embargo el hombre actual no muestra claridad en sus pasos hacia este anhelo, lo que circunda en muchos espacios son guerras en países y comunidades, busca de poderío en la política, corrupción y manipulación en los gobiernos, acceso insuficiente en la educación, desvinculación en la familia y sin sentido en muchos hombres.

En este artículo se analiza el concepto de paz y se proponen, desde el ámbito axiológico y educativo, herramientas que permitan ir a su encuentro. El objetivo del mismo es, ante la realidad caótica, perfilar el establecimiento de diferentes acepciones de lo que es la paz y distinguirlo del proceso de valoración que sigue el ser humanos para asumir alguna de estas concepciones. Se persigue aclarar que varios referentes asumidos socialmente, más que valores son normas o reglas bajo las cuales se juzga el entorno. En esta disertación se propone la educación como el medio que puede atender la urgencia de justicia, de paz y de comprensión en nuestras sociedades. 


\section{Abstract}

The value peace more than sought by the human being is a desired situation in humanity, but the modern man does not show clearly on his way to this desire, which in many surrounding areas are wars in countries and communities, seeking power in politics, corruption and government manipulation, insufficient access to education, disengagement in the family and meaningless in many men.

This article discusses the concept of peace and proposes, from within the axiological and educational tools to go to meet him. The objective is, given the chaotic reality, outline the establishment of different meanings of what peace is and distinguish it from the valuation process that follows the human being to take any of these conceptions. It is intended to clarify several references made socially rather than values are standards or rules under which judges the environment. In this dissertation proposes education as the medium that can meet the urgent need for justice, peace and understanding in our societies.

Palabras clave: Paz, valor, valoración, jerarquía, educación

Key Words: Paz, value, assessment, hierarchy, education

Doctora en Educación, Maestra en Educación Familiar, Licenciada en Filosofía. Docente-Investigadora de la Universidad La Salle Pachuca. efragoso@lasallep.edu.mx 


\section{Introducción}

Mientras escribo este documento una realidad doliente nos circunda, hay en este momento ejércitos lanzando bombas en pueblos indefensos, familias sufriendo hambre, padres que buscan un empleo ya no digamos digno, un empleo (a veces de explotación) que les dé hoy recursos para llevar alivio a su casa, ancianos sumidos en una violenta soledad, familias con relaciones dañinas que les son cotidianas. Tenemos que movernos de nuestra violencia habitual y abrir caminos para la comprensión de quiénes somos, el entendimiento hacia el otro - el diferente- a una acción que establezca una paz real. En la era de la globalización, entendiéndola no desde su base neoliberal basada en la economía dominante, sino desde este ámbito de paz como la iniciativa de un mundo sin fronteras, donde hay apertura a la pluralidad y una lucha sí, pero por una unidad en la diversidad.

Para el ser humano siempre han existido cosas valiosas: el bien, la verdad, la belleza, la felicidad, la virtud o la paz, entre otros. Sin embargo, el criterio para estimar ha variado a través de los tiempos. Se puede apreciar de acuerdo a criterios estéticos, esquemas sociales, costumbres, principios éticos o, en otros términos, como en la actualidad el económico (Ramonet,2008:1). En las sociedades consumistas, como son las actuales, el enfoque que se le da a los objetos o las acciones es economicista y a pesar de que algo no sea realmente valioso, por ser costoso, se le asigna un gran valor. Esto se debe a que no todas las personas profundizan en el concepto de valores, lo usan sin fundamento y esto produce confusiones en el proceso de valoración de las cosas, las personas o las acciones que realizan los seres humanos.

De ello parte el objetivo de este artículo, ante esta realidad caótica se perfila el establecimiento de un análisis que puntualice las diferentes acepciones de lo que es el valor de la paz y distinguirlo del proceso de valoración que sigue el ser humanos para asumir alguna de estas concepciones. Se persigue aclarar que varios referentes asumidos socialmente, más que valores son normas o reglas bajo las cuales se juzga el entorno. En esta disertación se propone la educación como el medio que puede atender la urgencia de justicia, de paz, de comprensión. 


\section{Origen de la falsa valoración}

Buscamos la felicidad en el mundo que nos rodea. Creemos que encontraremos la paz cuando tengamos la casa cómoda, la pareja perfecta, los mejores hijos o el trabajo ideal, nos afianzamos a que entonces habremos finalmente alcanzado la paz, y vivimos tratando de alcanzar aquello. Estamos buscando en el lugar equivocado. La paz no la encontraremos en el olvido de nosotros mismos, sentados inconscientes frente a la televisión, la paz la encontraremos cuando nos redescubramos. Esto se puede simbolizar:

"Había una vez una anciana que buscaba algo en medio de la noche. Se encontraba bajo un farol buscando algo en el pavimento, en la calle, en el cesto para basura y en todo lugar bajo el farol, pero no podía encontrar lo que estaba buscando. Un grupo de jóvenes pasó caminando. Uno de ellos le preguntó a la anciana: "Abuela, ¿qué está buscando?, ¿podemos ayudarla, abuela?" La anciana respondió: "Estoy buscando una aguja de coser. La perdí. Por favor ayúdenme a encontrarla." Los jóvenes ayudaron a la anciana a buscar la aguja de coser por todos lados, pero no pudieron hallarla. Entonces, uno de los jóvenes se acercó a la anciana y le preguntó: "Abuela, por favor díganos donde dejó caer la aguja, así sabremos por dónde empezar". La anciana respondió "Estaba cosiendo en mi habitación y dejé caer la aguja. Pero mi habitación es obscura y no puedo ver con claridad. En cambio, aquí hay mucha luz y puedo ver claramente, por eso la estoy buscando aquí." Cuando los jóvenes oyeron esto rieron y le dijeron a la anciana: "Abuela, tiene que buscar la aguja donde la perdió. No la hallará en ningún otro lado." (Jumsai \& Burrows, 2000: 27-28).

Eso es lo que nos pasa en la búsqueda de la paz. La estamos buscando en el lugar equivocado. No es a la luz del farol de la calle, sino en la oscuridad de nuestra habitación donde se encuentra la paz perdida. Para recobrar la paz intentamos diversos métodos: la acumulación de riqueza, el mantenimiento de la salud, el dominio del conocimiento, el cultivo de las artes. Pero éstos no son fundamentales (Sathya Sai, 1990: 85). 


\section{El camino aquí propuesto es el cultivo de una educación en valores}

El camino es atendido desde la Axiología, ciencia que estudia los valores, denominada también teoría de los valores porque comprende, por una parte, el estudio de la esencia y naturaleza de los valores y por otra; las valoraciones o juicios de valor. Es una ciencia que aparece en el siglo XIX y surge de la inquietud por lograr la sistematización de los valores. Es una rama de la metafísica dentro del ámbito de la filosofía, y posee la tarea de profundizar en la naturaleza de los entes. La axiología se relaciona con la ética porque da el fundamento valoral a las virtudes - los valores operativos en la conducta de los individuos- y con las demás ciencias, porque todas necesitan partir de determinados valores para plantear sus postulados.

Para López de Llergo (2000: 45) la axiología es una rama de la metafísica ---- proviene del griego axiós = valioso, estimable, digno de ser honrado y del logos que significa palabra, estudio o ciencia; así, la axiología es la ciencia -- que estudia los valores o teorías de los valores, profundiza por un lado la esencia del valor, la clasificación de los valores y el proceso de valoración (o juicios de valor).

\section{No hay una sola corriente axiológica, proponemos la escuela del realismo de los valores}

Las valoraciones son producto de cambios y transformaciones a lo largo de la historia. Surgen con un especial significado y cambian o desaparecen en las distintas épocas. Por ejemplo en la actualidad se defiende la igualdad de oportunidades para el desarrollo tanto de la mujer como del hombre, pero no se podría enseñar a las personas del mundo antiguo esta concepción tal y como se comprende hoy en día. Es precisamente el significado social que se atribuye a los "valores" uno de los factores que influye para diferenciar los valores tradicionales, aquellos que guiaron a la sociedad en el pasado, generalmente referidos a costumbres culturales o principios religiosos, y los valores modernos, los que comparten las personas de la sociedad actual.

De acuerdo al concepto de valor que se postula surgen diferentes corrientes axiológicas 1. Escuela Neokantiana ${ }^{1}$. 2. Escuela austriaca y de Praga $^{2}$. 3. Escuela existencialista ${ }^{3}$. 4. Escuela fenoménica ${ }^{4}$. 5. Escuela del realismo de los valores ${ }^{5}$. 
1 Esta escuela se apoya en la postura ética kantiana que se expone La forma de la conducta humana es el imperativo categórico que esta impreso en toda conciencia y que se enuncia así: "Obra de tal manera que la máxima de tu voluntad pueda valer para siempre como un principio de una legislación universal" (Kant, 1961: 37). Para los neokantianos los valores son principios, normas o ideales, se encuentran fuera de la conciencia, se dividen en relativos y absolutos. Asimismo y consideran que la cultura es un proceso de conquista de los valores.

2 Esta es una postura subjetivista que da primacía a la vivencia o las percepciones del sujeto, exalta la experiencia y la razón desde la única realidad existente del yo, por lo que se le conoce también como psicologismo lógico. Aquí el valor se considera en función del sujeto valorante, esto es, los valores se dan en el sujeto en función de la postura que adopte frente al objeto. Los valores se ubican en el agrado, el deseo o en la emotividad del sujeto. Dividen los valores en absolutos o impersonales y en relativos o según cada persona. La postura subjetivista es muy frecuente en la actualidad. Cuando se cree que la única verdad es la que proviene de la experiencia personal o de los razonamientos de cada uno, sin tener en cuenta la realidad; o se asume una defensa de lo que se considera valioso incurriendo en subjetivismos.

3 Se trata de un enfoque sobre la reflexión de la experiencia humana de la existencia. Lleva al ser humano a afrontar su existencia con una responsabilidad total, que se traduce en

angustia cuando hay que elegir y hace referencia a un sistema de valores sin connotación sobrenatural.

La escuela parte de la fenomenología como experiencia de la conciencia, la clave del método consiste obtener la esencia del fenómeno a partir del fenómeno mismo. La fenomenología es un esfuerzo por volver a las cosas mismas, por llegar a la realidad en su integridad, eliminando los ídolos, los prejuicios y los condicionamientos. El fenómeno es la manifestación de la realidad en la conciencia

5 El realismo tiene como postulado fundante que lo real es lo que tiene ser. Sostiene que los valores están enraizados en el ser, por lo que son algo real en identidad al ser. Es una

De estas corrientes, la visión subjetivista considera que los valores no son reales, no valen en sí mismos, sino que son las personas quienes les otorgan un determinado valor, dependiendo del agrado o desagrado que producen. Desde esta perspectiva, los valores son subjetivos, dependen de la impresión personal del ser humano. La escuela neokantiana afirma que el valor es, ante todo, una idea. Se diferencia lo que es valioso, de lo que no lo es, dependiendo de las ideas o conceptos generales que comparten las personas. Algunos autores indican que no son concretos, no se encuentran en el mundo sensible y objetivo, sino en el pensamiento y en la mente; ahí es donde los valores se aprehenden, cobran forma y significado. En este relativismo, cada quien crea su propia jerarquía y defiende sus propios valores, en esta línea de pensamiento sí se podría hablar de que los valores de los hijos ya no concuerdan con los de los padres.

En el ámbito objetivista, en cambio, la concepción del valor parte de la observación de la realidad y coloca al valor en el ser de los entes (todo lo que existe). 
Desde esta postura la realidad es lo que es, independientemente de la opinión de quien la conozca. Dentro de esta corriente encontramos la escuela realista que considera que los valores son objetivos; valen independientemente de las cosas y de las estimaciones de las personas. Así, aunque todos seamos injustos, la justicia sigue teniendo valor.

En este trabajo se afirma que los valores tienen realidad, son reales porque se apoyan en el ser de los entes; es decir, en su naturaleza misma; sin embargo, también se acepta que existe en su conocimiento, un proceso de valoración, que es la forma en la que el hombre capta ese valor ya existente, el cual se produce de manera subjetiva, es decir, partiendo de lo que el sujeto es. Esto bien entendido no es lo mismo que subjetivista (Fragoso, 2006:103)

\section{Concepto de valor}

Desde el punto de vista etimológico valor proviene de valere (Gómez de Silva 2001:708), ser fuerte, ser potente. Y esta potencia es lo que permite que cada ser se manifieste, se realice como tal ser.

teoría de los valores enraizada en una metafísica general. Su método es la dialéctica. Los valores se configuran en el hombre en función del deber y del ser. Como la conciencia humana es actividad y libertad, tienen significados para el hombre que puede apropiarse de los valores por la experiencia, y realizarlos en la vida. Los valores solo tienen valor para las personas pero ellas no los emanan. La búsqueda de los valores tiene un sentido finalístico.

López de Llergo (2000:23) explica, que un valor es una perfección real o posible, real porque está enraizada en el ser de todo lo que existe y posible porque está potencialmente presente y puede actualizarse. Esta perfección procede de la naturaleza que se encuentra en el ser y en la razón de ser. El ser es el soporte de los valores pero también la razón de ser, su misión, lo que le da sentido en la realidad. Desde esta perspectiva los valores son inamovibles; cada ser no cambia su esencia mientras permanece lo que es. 
Lo que es perfecto es admirado por los hombres, por ejemplo la armonía de una pieza musical o la contemplación del oleaje rítmico del mar, pero esto y todo lo demás valioso, no lo es porque el hombre lo ha valorado o no, sino porque ya tenía en sí mismo ese valor; el hombre no se lo dio, sólo fue capaz de captarlo y en consecuencia apreciarlo.

El valor emana de la esencia de las cosas, es algo sin lo cual el ser no podría ser lo que es, o no existiría como tal. El valor permite identificar cada cosa, es algo que no puede cambiar en cada ser, es aquello que le brinda a cada ser lo que es. Si se tienen diferentes tipos de zapatos, ellos poseen color, forma, medida, precio, textura, diferentes. Si se pone fuera todos los atributos que hacen distintos a los zapatos, sólo resta su función: es proteger el pie al caminar. Ese es el valor de los zapatos. De la misma manera, el valor del fuego es la capacidad de calentar y el valor de la sal es, ser salada; así si no sala, se afirma que simplemente no es sal.

Al estar los valores en la esencia de los seres, la tarea del hombre es descubrirlos, en el sentido etimológico de descubrir, es decir, quitarle la cubierta, y así comprender que la naturaleza solo actúa bajo su misma ley, una ley perfecta e inquebrantable llamada logos, y el hombre siendo parte de esa naturaleza, no puede más que estar hecho para entenderla, para armonizar con ella, en ese sentido reflexionarla, razonarla e irla haciendo suya, aprenderla y aprehenderla; y esto es desentrañar su logos, su valor, lo que la hace ser lo que es.

El concepto de valor abarca contenidos y significados diferentes y ha sido abordado desde diversas perspectivas y teorías. En sentido humanista, se entiende por valor del hombre, lo que hace que éste sea tal (hombre), es decir sin lo cual perdería la humanidad o parte de ella. El valor se refiere a una excelencia o a una perfección. En este sentido, la práctica del valor desarrolla la humanidad de la persona, mientras que el contravalor lo despoja de esa cualidad (Vásquez, 1999: 3).

Desde un punto de vista socio-educativo, los valores son considerados referentes, pautas o abstracciones que orientan el comportamiento humano hacia la transformación social y la realización de la persona. Son guías que dan determinada orientación a la conducta y a la vida de cada individuo y de cada grupo social. 


\section{Proceso de valoración en el humano}

La relación que el hombre establece con el mundo se gesta a partir de la valía que éste establece. En este sentido hay dos actos importantes en el proceso de valoración de los entes: primero que se capte la perfección, y después el que merezca ser estimada (López de Llergo, 2000: capítulo V).

Se llaman valores a las perfecciones, aquello que es bueno; y las virtudes son las operaciones por las cuales son llevados a cabo. El ser humano es de una naturaleza precaria por lo cual debe realizar actos que lo perfeccionen de un modo integral para sobreponerse a su carencia natural de plenitud.

La educación en valores, como señala López de Llergo (2000:109) se concibe como una orientación a la inteligencia con apoyo de la voluntad, para profundizar y respetar los valores que se encuentran en lo más íntimo de la naturaleza humana y en el ser de otras criaturas. El valor actualiza el ejercicio de la inteligencia, captamos el valor al conocer. En un primer momento el hombre conoce la realidad, la comprende y la contempla, ahí descubre su valor. La valoración ejercita el hacer de los juicios. Al valorar se volca uno sobre las cosas: las ordena, las jerarquiza y las clasifica. Los valores dan luz a la inteligencia para que guíe a la voluntad (segundo momento) y se hacen vida cuando el ser humano adquiere las virtudes. La educación en valores debe ser el punto de partida de toda propuesta educativa y la educación en virtudes el punto de llegada. La voluntad desarrolla del dominio sobre sí mismo lo que le permitirá desarrollar las virtudes, los hábitos buenos que conducen su actuar.

Valoración es el acto de valorar o sea, evaluar, estimar, apreciar. Valorar es descubrir el valor de algo. Es captar y expresar a mi manera, la perfección de la realidad. Es captar lo objetivo pero al modo personal siempre que incluya el respeto a la realidad. Es un planteamiento subjetivo pero no subjetivista.

¿Cómo valora el ser humano? El proceso de valoración del ser humano incluye una compleja serie de condiciones intelectuales y afectivas; en él interviene la inteligencia que estima y valora lo singular a partir de los datos sensibles por lo tanto, suponen: la toma de decisiones, la estimación y la actuación. Las personas valoran al preferir, al estimar, al elegir unas cosas en lugar de otras, al formular metas y propósitos personales. 
Las valoraciones se expresan mediante creencias, intereses, sentimientos, convicciones, actitudes, juicios de valor y acciones.

Al valorar algo, se reflexiona sobre eso para conocerlo y comprenderlo en sus características y capacidades, se adquiere experiencia, vivencia propia de lo captado y la inteligencia completa el proceso en un conocimiento racional que produce ideas y conceptos sobre el ente, se capta la esencia, lo propio del ser. Se captan los valores encerrados en la realidad y se producen juicios y con ellos la valoración.

Es un proceso subjetivo de captación de la realidad objetiva. Depende de las cualidades del sujeto que valora y no sólo de lo valorado. Y es donde puede surgir un error de valoración. Captar la mejor razón de ser depende de la personalidad y educación del sujeto.

\section{Jerarquía y jerarquización de valores}

¿Qué hace que algo sea valioso?, la jerarquía misma de la naturaleza nos muestra la jerarquía de valores (Cfr. López de LLergo, 2000).

Se le puede dar a un valor mayor importancia o no, esto ya es una valoración personal que depende de la forma en que cada quien construye la realidad, pero no se ha de confundir esto, con lo que realmente es la escala de los valores, sino se ha de estar consciente de que es sólo la percepción particular de un individuo.

Para captar la escala que tiene un valor no hay que ordenarlo de acuerdo a los propios principios porque entonces sólo se está jerarquizando o creando una jerarquía propia, pero si se quiere ser objetivo y captar el valor en su real interrelación con los demás, se ha de apegar a la naturaleza misma, quien muestra la natural jerarquía de los seres, de cuya esencia, se desprenden su valores.

El valor se infiere desde el ser del ente; se manifieste o no, ahí se encuentra: "puede decirse que el ser es el primer valor, del que se seguirán los demás valores de ese ente, según su naturaleza. Así pues, los valores sólo se dan en la realidad que es o que opera" (López de Llergo, 2000:23).

Pero en todo esto hay el peligro de ser subjetivista y decir "ese es mi valor no es el tuyo" y esa es una de las razones por las que en la sociedad y en los hogares se entablan discusiones infundadas intergeneracionales. 
La idea que se tiene comúnmente de los valores es que son un constructo, que se ha ido creando a partir de las experiencias, lo cual es erróneo. En tanto más se apegue la interpretación de la realidad al conocimiento que se tiene de la esencia de las cosas, más correcta será la concepción y mientras más se apegue a los supuestos sociales, la ideología de un lugar o elucubraciones personales, más alejado se estará de la verdad.

No es ni el maestro por tener conocimientos, ni el padre por tener más experiencia, ni el cura por su conocimiento de la sagrada escritura, quienes determinan lo que es un valor o lo valioso; es la misma naturaleza con sus propias leyes quien muestra la escala de los valores. Si los individuos en el mundo o los padres e hijos en cada hogar se apegaran a estos conocimientos, muchos conflictos terminarían.

\section{Valor de la paz}

¿Por qué no encontramos la felicidad tan perseguida? Martha Washington afirma: "La mayor parte de nuestra felicidad o miseria depende de nuestras disposiciones y no de nuestras circunstancias" (Helen Buss, 1998: 120) Alrededor de nosotros pueden ocurrir acontecimientos agradables o desagradables, alguien quizá nos insulte o nos halague, la casa puede estar llena de comodidades o ser una habitación pobre, pero la felicidad podrá ser experimentada, sólo si estamos dispuestos a ello, si hemos desarrollado nuestra capacidad para vivir en el mundo, sin que el mundo viva en nosotros.

En el universo entero todo es dual, hay noche y día, oscuridad y luz, muerte y vida, dolor y placer. Un sabio consejo ante la contrastante dualidad de la vida es recordarnos constantemente "Ya pasará". Aferrarnos a un extremo de las cosas es el método más seguro de vivir en desequilibrio. Cuando recibimos una moneda la aceptamos como es, con sus dos caras. No insistimos en "sólo me gusta el águila". Así también la vida debiéramos aceptarla como es, con sus dos caras de placer y dolor, de éxitos y fracasos. Y en realidad, si vamos a un análisis más profundo del entorno podemos no quedarnos con esta visión ontológica que nos heredó Parménides del ser y no sé y llegaríamos a vislumbrar que ambos elementos no son opuestos, sino diferentes aspectos del mismo ser, así frío y caliente no se oponen; son la expresión de dos tipos de temperatura distinta, hombre y mujer no se oponen; son complementarios. 
La estrategia central para vivir la paz la encontramos en una fuerza interna, explicada de la siguiente manera en la Cátedra UNESCO de Educación para la Paz, en la que Pascual Anaída afirma que "de cara a las injusticias, nos eduquemos en el valor moral para tomar riesgos y emprender acciones de naturaleza no violenta. Es decir, que nos formemos en aquella fortaleza del alma o voluntad del espíritu que posibilita la única y auténtica fuerza de verdad y vida: la no violencia" (Pascual, 2008: 123).

No obstante, hoy parece olvidada esta acción no violenta que era ya expresada en el legado de paz que nos dejaron personajes como Gandhi, Luther King o Daisaku Ikeda; pero en contradicción a sus propuestas se genera más armamento, subsisten las guerras y la violencia toca continuamente nuestras vidas permaneciendo en una parte de la humanidad un fascinación por la violencia, hacia la cual afirma David Reisman de la Universidad de Harvard "una vez la violencia se convierte en una adicción, la personalidad cambia: los impulsos más generosos quedan reprimidos; la gente se brutaliza y declara que la sociedad los ha hecho de esta manera" (Reisman, en Pascual, 2008:123)

Todos decimos "Yo quiero paz", pero como afirmaba anteriormente, parece que la buscamos en el lugar equivocado, no en las próximas vacaciones o cuando me suban el sueldo... La paz la encontraremos cuando nos redescubramos; para llegar nosotros mismos solo existen dos obstáculos, lo que creo ser y lo que deseo tener. Al analizar la sentencia "Yo quiero paz", Jumsai y Burrows explican que para alcanzar esta paz tan deseada, solo basta eliminar el "yo" y el "quiero" (Cfr. Jumsai y Burrows , 2000), ¿Por qué?:

El quiero son los deseos que nos empujan a vivir el tener y no, el ser. El deseo es como una hoguera insaciable, nos exige leños una y otra vez. Cuando tenemos la casa, ya queremos los muebles, cuando tenemos los muebles ya queremos el auto, cuando tenemos el auto ya queremos el viaje a aquel lugar hermoso y así nos pasamos la vida trabajando para satisfacer nuestros deseos y no tenemos tiempo para Ser.

"Yo" es el ego. Cuando alguien nos critica, el sonido llega a nuestros oídos y choca con el yo; entonces el yo se enfada y se enoja. Cuando nos libremos del yo, el sonido de la crítica no tendrá con qué chocar y permaneceremos en calma. Al yo le gusta compararse con otros (yo soy mejor, más rico, más hermoso, etc.) lo cual crea división en lugar de unidad. 
A veces se compara de un modo negativo (yo no soy tan rico o tan inteligente como él) entonces se siente celoso, se enoja y se incomoda. El yo destruye la paz, es solo lo que creemos ser, nuestras múltiples máscaras, por lo que tenemos que librarnos de él para recuperar la paz.

Abandonando la identificación con nuestro ego y estableciendo un límite para nuestros deseos no habrá razón para sufrir o gozar por lo que ocurre a nuestro alrededor, simplemente serán sucesos, experiencias vividas por nuestro Ser, los sentimientos estarán quietos para permitir que vibren bajo la energía de la paz.

\section{Conclusión}

Ante la violencia generada a diario en nuestras vidas, hemos de reflexionar que nuestros verdaderos enemigos son los estados mentales negativos (ira, lujuria, envidia, codicia, orgullo, odio, apego, temor, ansiedad). Todos ellos están almacenados en el subconsciente y tenemos que aprender a vencerlos y no permitirles emerger a la mente consciente. Para ello no sirve tratar de reprimir nuestras emociones. Es como un globo: si lo apretamos, habrá más presión del aire interno. Y si continuamos apretando gradualmente, la presión se volverá cada vez mayor, si apretamos más aún, la presión quizás llegue al límite y el globo reviente. Del mismo modo, cuando reprimimos nuestras emociones, tenemos mayor presión, lo que crea mayor tensión interna.

¿Cómo podemos controlar nuestras emociones y no permitirles que emerjan en nuestra mente consiente? La libertad progresiva y el imperio sobre la propia mente están perfectamente dentro de las posibilidades de todo aquel que tenga la fe y la voluntad de emprender esta conquista. Las posibilidades del ser mental no son limitadas; cada quien puede ser el libre testigo y el amo en su propia casa.

Afirma Aurobindo, el primer paso es tener una mente sosegada. El silencio es un paso ulterior, pero es necesario obtener previamente el sosiego. Y por mente sosegada entiendo una consciencia mental interior que ve los pensamientos acercarse a ella y moverse en torno, pero no se siente a sí misma pensando, ni se identifica con los pensamientos, ni los considera suyos. 
Los pensamientos y los movimientos mentales pueden pasar a través de esta consciencia mental interior tal como los caminantes aparecen procedentes de cualquier parte y pasan a través de una campiña silenciosa; la mente sosegada los observa o ni siquiera se toma la molestia de observarlos, pero en ningún caso participa en la acción o pierde su tranquilidad. El segundo paso es el silencio, que es más que el sosiego. Puede obtenerse desterrando completamente los pensamientos de la mente interior, manteniéndolos mudos o completamente aparte. Pero se establece con mayor facilidad con una práctica continua de silencio interno o meditación; cuando sucede así, se percibe cómo desciende el silencio, cómo penetra y ocupa o rodea la consciencia personal, que tiende entonces a sumergirse en el vasto silencio impersonal (Cfr. Aurobindo,1998).

Otra técnica para la vivencia de la paz es establecer continuamente pensamientos positivos, acciones rectas, decisiones adecuadas. Sin embargo, el poder más grande que confiere paz es el amor, el amor puro y desinteresado que sentimos por los demás."Es la ley del amor la que rige a la humanidad. Si la violencia, es decir el odio, nos rigiera; hace mucho tiempo estaríamos extintos. $Y$ sin embargo, la tragedia de todo esto es que las así llamadas personas y naciones civilizadas se conducen así mismas como si la base de la sociedad fuera la violencia" (Gandhi, 1951 en Pascual, 2008: 123). La no violencia exige acatar el llamado de la voz interior a una resistencia pacífica pero nunca pasiva. Sin embargo, "La no violencia no puede ser predicada: Tiene que ser practicada”.(Gandhi, 1948 en Pascual, 2008:124).

"La no violencia-en-acción supone una revisión profunda de nuestras vidas. Más aún, supone una inquebrantable voluntad del espíritu para permanecer fieles a los fines de una paz integral y a sus medios no violentos. Precisamente de esta paz indómita es que surgen visiones como la que hoy nos convoca a encaminarnos... hacia una cultura de paz." (Pascual, 2008:124).

El querer sustituir la intolerancia, la injusticia, la pobreza, crimen, la intimidación, el fanatismo, y todas las demás formas de violencia por la paz es un reto que huele a sueño, porque es un meta política, educativa, psicológica, sociológica, antropológica, estética, filosófica, espiritual, en medio de un proceso de globalización acelerado que abre brechas insólitas. 
Tenemos que participar del anhelo universal por vivir en paz y en forma sostenible con la biosfera, en armonía con todos los pueblos del mundo, con todas las formas de existencia; y participar es, formar parte de, es decir, practicar de manera inquebrantable los valores de una cultura de paz individual y colectiva.

\section{Fuentes de Consulta.}

actitudes.

ALCÁNTARA, José Antonio. (1998). Cómo educar las

Barcelona, España: Ediciones Ceac

Fundación

AUROBINDO (1998) Guía del yoga interior, Barcelona:

Centro Sri Aurobindo,

MiNos.

Corominas Fernando. (1993). Educar la voluntad. México:

-

FRAGOSO Fernández, Esther. (2006). "¿Son los valores subjetivos u objetivos? Diferenciación entre lo que es un valor es si y el proceso de valoración" en Xihmai Vol 02, ISSN 18706703.

- GIMENO Sacristán J. y Pérez Gómez A.I. (1998). Comprender y transformar la enseñanza. Madrid, España: Ėdiciones Morata.

GÓMEZ de silva, Guido. (2001), Diccionario etimológico de la lengua Española. México: Fondo de Cultura Éconómica

HELEN Buss Mitchell (1998) Raíces de la sabiduría, México: International Thompson Editores

Tomo III.

HIRSCH, Alder Ana (2005) Educación y valores.

México: GERNIKA

Art Ong Na Ayudhya. y Loraine, B. (2000). Amor:
Corazón de la enseñanza. Programa de Educación en Valores Humanos

JUMSAI, Sathya Sai. México: Ediciones Sai Ram.

- LÓPEZ de Llergo Ana Teresa. (2000). Valores, valoraciones y virtudes. Metafísica de los valores. México: CECSA.

Buenos Aires: Errepar

MONTESSORI, María. (1998) Educación y paz,

PASCUAL Morán, Anaída. (2008),"Investigación, educación y acción noviolenta: La única vía hacia una Cultura de Paz"” en Cátedra UNESCO de Educación para la Paz. Educando para la Paz en y desde la Universidad., Puerto Rico: Universidad de Puerto Rico, pp 121-136.

RAMONET, Ignacio (2008),"El nuevo orden mundial" 
en Cátedra UNESCO de Educación para la Paz. Educando para la Paz en y desde la Universidad., Puerto Rico: Universidad de Puerto Rico, pp 1-12.

- SATHYA Sai, (1990). Sathya la senda de la verdad. México: Ediciones Sai Ram. 
